\title{
Integer superspin supercurrents of matter supermultiplets
}

\author{
I.L. Buchbinder, ${ }^{a, b}$ S. James Gates Jr. ${ }^{c}$ and K. Koutrolikos ${ }^{c}$ \\ ${ }^{a}$ Department of Theoretical Physics, Tomsk State Pedagogical University, \\ Tomsk 634041, Russia \\ ${ }^{b}$ National Research Tomsk State University, \\ Tomsk 634050, Russia \\ ${ }^{c}$ Department of Physics, Brown University, \\ Box 1843, 182 Hope Street, Barus 6 Holley 545, Providence, RI 02912, U.S.A. \\ E-mail: joseph@tspu.edu.ru, sylvester_gates@brown.edu, \\ konstantinos_koutrolikos@brown.edu
}

ABSTRACT: In recent papers $[18,21]$ we demonstrated that consistent and non-trivial linear transformations of matter supermultiplets generate half-integer superspin supercurrents and the cubic interactions between matter and half-integer superspin supermultiplets. In this work we show that consistent and non-trivial antilinear transformations of matter superfields lead to the construction of integer superspin supercurrents and the cubic interactions between mater and integer superspin supermultiplets. Applying Noether's method to these transformations, we find new integer superspin supercurrents for the case of a free massless chiral superfield. Furthermore, we use them to find new integer superspin supercurrent multiplets for a massive chiral superfield and a chiral superfield with a linear superpotential. Also various selection rules for such interactions are found.

KEywords: Higher Spin Symmetry, Superspaces, Supersymmetric Effective Theories

ARXIV EPRINT: 1811.12858 


\section{Contents}

1 Introduction 1

2 Gauge invariant interacting theories of matter with gauge fields $\quad 2$

3 Anti-linear transformation of the chiral superfield 5

4 Conformal integer superspin supercurrents for free massless chiral $\quad 7$

5 Poincaré integer superspin supercurrents for free massless chiral $\quad 11$

6 Integer superspin supercurrent multiplet beyond free, massless, chiral 11

$\begin{array}{lll}6.1 & \text { Free chiral with linear superpotential } & 12\end{array}$

$\begin{array}{lll}6.2 & \text { Free massive chiral } & 13\end{array}$

$\begin{array}{lll}7 & \text { Summary and discussion } & 13\end{array}$

\section{Introduction}

For non-supersymmetric theories there is a plethora of well-known results on the topic of higher spin conserved currents [1-10] and higher spin cubic interactions [11-16]. Recently some of these results have been extended to supersymmetric theories. In a series of papers [17-24] a variety of supersymmetric, higher spin, currents have been constructed for miscellaneous matter and higher spin supermultiplets while the corresponding cubic interactions between matter and higher spin supermultiplets or between higher spin and higher spin supermultiplets have been discussed.

In most of these considerations, the multiplet of supercurrents were found by solving the appropriate conservation equations. However, for $[18,21]$ the foundation of the construction was the discovery of a linear non-trivial consistent, first order, higher spin transformation of matter superfields. Specifically, it was shown that the most general linear transformation of matter superfields, which is non-trivial and consistent with the various constraints of matter supermultiplets (chiral, or complex linear) is parametrized by terms that match the gauge symmetry of free, massless, half-integer superspin $[Y=s+1 / 2]$ supermultiplets $(s+1, s+1 / 2)$. The application of Noether's method to this kind of deformation lead us to the construction of higher spin supercurrents and higher spin supertraces which generate the cubic interactions of the various matter supermultiplets with the half-integer superspin supermultiplets. The construction is reminiscent of the way that linearized superdiffeorphisms lead to the construction of the supergravity supercurrent and supertrace of matter supermultiplets. Nevertheless, the absence of integer superspin $[Y=s]$ supermultiplets $(s+1 / 2, s)$ from the above consideration was intriguing. 
The purpose of this work is to find appropriate higher spin deformations of the matter superfields that lead via Noether's method to the construction of integer superspin supercurrents and generate the cubic interactions with free, massless integer superspin supermultiplets. We find that there exist non-trivial, antilinear transformations ${ }^{1}$ of the matter superfields that will generate these interactions. Specifically, we write the most general antilinear transformation for a chiral superfield, demand it to be non-trivial and compatible with the chiral constraint. The result is that parameters of the transformation have the same structure with the gauge symmetry of free, massless integer superspin supermultiplets. That means by performing Noether's method, we can construct the integer superspin supercurrent multiplet (includes the supercurrent $\mathcal{J}_{\alpha(s) \dot{\alpha}(s-1)}$ and the supertrace $\left.\mathcal{T}_{\alpha(s-1) \dot{\alpha}(s-1)}\right)$ and generate the cubic interactions between the free, massless chiral superfield and the integer superspin supermultiplets $(s+1 / 2, s)$. The results are extended to the case of a free, massive chiral and a free chiral with linear superpotential.

It is known that any $\mathcal{N}=1$ supersymmetric matter theory can be consistently coupled to supergravity with the help of the gravitational superfield. For that case the calculation of the conserved supercurrent is straightforward. One has to take the functional derivative of the interacting action with respect the gravitational superfield (see e.g. [25, 26]). However, this procedure is not applicable for higher spin theory because we do not know the fully interacting theory at present. The only alternative option we have is to follow Noether's method in order to construct directly the higher spin supercurrent multiplet of the theory.

The paper is organized as follows. Section 2 reviews the philosophy and the details of Noether's method as well as it provides to the non-expert reader the essentials for the description of $4 D, \mathcal{N}=1$ arbitrary integer superspin supermultiplets for both the Poincaré and conformal cases. In section 3, we consider first order transformations (in the spirit of Noether's method) of the chiral superfield which are antilinear and demonstrate the fixing of their parameters by requiring them to be consistent with the chiral constraint of the superfield and non-trivial. Sections 4 and 5 consider the case of a single, free, massless chiral superfield and derive the conformal and Poincaré supercurrents respectively using the deformations of section 3. In section 6 , we extend this results for the two case of a free, massive chiral superfield and a free chiral with linear superpotential. In the last section 7 , we discuss and summarize our results.

\section{Gauge invariant interacting theories of matter with gauge fields}

It is a fact of physics that a manifestly Lorentz invariant and local description of massless degrees of freedom with spin greater than $1 / 2$ requires the identification of various field configurations (gauge symmetries). As we transition perturbatively from free theories to interacting ones, the notion of this identification has to be re-examined in every step. This can be done systematically by expanding the action $S[\phi, h]$ and the transformation of all

\footnotetext{
${ }^{1} \mathrm{~A}$ map $f: V \rightarrow W$ from one complex vector space $V$ to another $W$ is called antilinear if $f(a u+b v)=$ $a^{*} f(u)+b^{*} f(v)$ where $a, b$ are complex numbers and $u, v$ are elements of $V$. This is equivalent to a linear map from $V$ to the complex conjugate vector space $\bar{W}$. The transformations we consider have this property, they are linear in the complex conjugate of the superfield.
} 
fields in a power series of a coupling constant $g$.

$$
\begin{aligned}
S[\phi, h] & =S_{0}[\phi]+g S_{1}[\phi, h]+g^{2} S_{2}[\phi, h]+\ldots, \\
\delta \phi & =0+g \delta_{1} \phi+g^{2} \delta_{2} \phi+\ldots \\
\delta h & =\delta_{0} h+g \delta_{1} h+g^{2} \delta_{2} h+\ldots
\end{aligned}
$$

In the above expressions we consider the interaction of a set of matter fields represented by $\phi$ with a set of gauge fields represented by $h$. Matter fields do not have a zeroth order gauge transformation $\left(\delta_{0} \phi=0\right)$, whereas gauge fields do $\left(\delta_{0} h \neq 0\right)$. The terms $S_{i}[\phi, h]$ correspond to interaction terms of order $i+2$ in the number of fields and $\delta_{i}$ is the part of transformation with terms of order $i$ in the number of fields. The invariance of the theory under these transformations can be studied iteratively, order by order. For cubic order terms $S_{1}[\phi, h]$ we get

$$
\int\left\{\frac{\delta S_{0}}{\delta \phi} \delta_{1} \phi+\frac{\delta S_{1}}{\delta h} \delta_{0} h\right\}=0
$$

The above expression is a symbolic one. There are a number of hidden summations over 'repeated fields' ${ }^{2}$ which are suppressed and represented by the integral sign. This invariance condition (up to cubic terms) makes very clear the importance of the first order correction in the transformation of the matter fields, $\delta_{1} \phi$. The starting action $S_{0}$ is known and the zeroth order transformations of gauge fields are also known. Hence, in order to find a consistent set of non-trivial cubic interactions $S_{1}[\phi, h]$ we must find a non-trivial $\delta_{1} \phi$. In this consideration, trivial interactions and trivial transformations are the ones that can be absorbed by an appropriate redefinition of the fields or in other words they vanish under the consideration of the equations of motion.

Cubic interactions of a matter theory with gauge fields can be written in the form $j h$ where $j$ is a current constructed out of the matter fields which plays the role of the source. For these types of interactions, condition (2.4) takes the form

$$
\int\left\{\frac{\delta S_{0}}{\delta \phi} \delta_{1} \phi+j \delta_{0} h\right\}=0
$$

from which one can recover the conservation law of the current $j$ by using the equations of motion (up to the appropriate order, for this case it is $\frac{\delta S_{0}}{\delta \phi}=0$ ) and the structure of the gauge transformation of $h\left(\delta_{0} h=\partial \lambda\right)$.

In recent papers $[18,21]$, this approach has been used in order to construct conserved, higher spin supercurrents for the chiral $\left(\Phi, \overline{\mathrm{D}}_{\dot{\alpha}} \Phi=0\right)$ and complex linear $\left(\Sigma, \overline{\mathrm{D}}^{2} \Sigma=0\right)$ supermultiplets. In these papers we considered the most general, non-trivial, first order transformations $\delta_{1} \Phi, \delta_{1} \Sigma$ which depend linearly on $\Phi$ and $\Sigma$ respectively. These transformations are a higher spin extension of linearized superdiffeomorphism and like superdiffeomorphism generate interactions to supergravity supermultiplet $(2,3 / 2)$, they generate interactions to

\footnotetext{
${ }^{2}$ There is a summation over hidden external indices that count the number of matter and gauge fields, there is a summation over the hidden spacetime indices of the gauge fields and an integration over the spacetime coordinates.
} 
arbitrary higher spin supermultiplets of type $(s+1, s+1 / 2)$ (called half-integer superspin supermultiplets) for any non-negative integer $s$.

In this work, we explore the possibility of non-trivial, first order transformations that depend antilinearly on the matter superfield. In the following sections we will find that such transformations do exist and generate interactions to arbitrary higher spin supermultiplets of type $(s+1 / 2, s)$ (called integer superspin supermultiplets). We briefly remind the non-expert reader that the superspace Lagrangian description of free, massless, superPoincaré, arbitrary high $(s \geq 2)$, integer superspin supermultiplet involves a fermionic superfield $\Psi_{\alpha(s) \dot{\alpha}(s-1)}$ and a real bosonic superfield $V_{\alpha(s-1) \dot{\alpha}(s-1)}$ with the following zeroth order gauge transformations

$$
\begin{aligned}
\delta_{0} \Psi_{\alpha(s) \dot{\alpha}(s-1)} & =-\mathrm{D}^{2} L_{\alpha(s) \dot{\alpha}(s-1)}+\frac{1}{(s-1) !} \overline{\mathrm{D}}_{\left(\dot{\alpha}_{s-1}\right.} \Lambda_{\alpha(s) \dot{\alpha}(s-2))}, \\
\delta_{0} V_{\alpha(s-1) \dot{\alpha}(s-1)} & =\mathrm{D}^{\alpha_{s}} L_{\alpha(s) \dot{\alpha}(s-1)}+\overline{\mathrm{D}}^{\dot{\alpha}_{s}} \bar{L}_{\alpha(s-1) \dot{\alpha}(s)} .
\end{aligned}
$$

Off-shell, this supermultiplet carries $8 s^{2}+8 s+4$ bosonic and equal number of fermionic degrees for freedom. ${ }^{3}$ The physical ${ }^{4}$ (propagating) degrees of freedom are described by a field strength ${ }^{5}$ superfield $\mathcal{W}_{\alpha(2 s)}$

$$
\mathcal{W}_{\alpha(2 s)} \sim \overline{\mathrm{D}}^{2} \mathrm{D}_{\left(\alpha_{2 s}\right.} \partial_{\alpha_{2 s-1}} \dot{\alpha}_{s-1} \partial_{\alpha_{2 s-2}} \dot{\alpha}_{s-2} \ldots \partial_{\alpha_{s+1}}^{\dot{\alpha}_{1}} \Psi_{\alpha(s) \dot{\alpha}(s-1)}
$$

The super-field strength is chiral $\left(\overline{\mathrm{D}}_{\dot{\beta}} \mathcal{W}_{\alpha(2 s)}=0\right)$ and on-shell satisfies the following equation of motion:

$$
\mathrm{D}^{\beta} \mathcal{W}_{\beta \alpha(2 s-1)}=0
$$

There is also a super-conformal integer superspin supermultiplet. Its Lagrangian description is given in terms of the super-field strength $\mathcal{W}_{\alpha(2 s)}$. Similarly with the super-Poincaré case, the super-field strength can be expressed in terms of a prepotential $\Psi_{\alpha(s) \dot{\alpha}(s-1)}$ (as in (2.7)) whose gauge transformation saturates the maximum symmetry group of $\mathcal{W}_{\alpha(2 s)}$

$$
\delta_{0} \Psi_{\alpha(s) \dot{\alpha}(s-1)}=\frac{1}{s !} \mathrm{D}_{\left(\alpha_{s}\right.} \Xi_{\alpha(s-1)) \dot{\alpha}(s-1)}+\frac{1}{(s-1) !} \overline{\mathrm{D}}_{\left(\dot{\alpha}_{s-1}\right.} \Lambda_{\alpha(s) \dot{\alpha}(s-2))} .
$$

As is demonstrated in (2.5) the conservation law of the current (multiplet) is determined by the gauge transformation of the gauge fields. Similarly, one can use (2.6a) and (2.9) to extract the corresponding superspace conservation equations:

$$
\begin{array}{rlrlrl}
\text { Poincaré: } & \mathrm{D}^{2} \mathcal{J}_{\alpha(s) \dot{\alpha}(s-1)} & =\frac{1}{s !} \mathrm{D}_{\left(\alpha_{s}\right.} \mathcal{T}_{\alpha(s-1)) \dot{\alpha}(s-1)}, & & \overline{\mathrm{D}}^{\dot{\alpha}_{s-1}} \mathcal{J}_{\alpha(s) \dot{\alpha}(s-1)}=0, \\
\text { Conformal: } & \mathrm{D}^{\alpha_{s}} \mathcal{J}_{\alpha(s) \dot{\alpha}(s-1)}=0, & \overline{\mathrm{D}}^{\alpha_{s-1}} \mathcal{J}_{\alpha(s) \dot{\alpha}(s-1)}=0 .
\end{array}
$$

\footnotetext{
${ }^{3}$ A detailed counting of the off-shell degrees of freedom can be found in [36-38, 40].

${ }^{4}$ The on-shell degrees of freedom are the 2 helicities of spin $j=s+1 / 2$ and the two helicities of spin $j=s$.

${ }^{5}$ This is the simplest gauge invariant object that does not vanish on-shell.
} 
These are the superspace conservation equations for the integer superspin supercurrent $\mathcal{J}_{\alpha(s) \dot{\alpha}(s-1)}$ and supertrace $\mathcal{T}_{\alpha(s-1)) \dot{\alpha}(s-1)},{ }^{6}$ for the super-Poincaré and the super-conformal cases respectively.

For a detailed review of free, massless, supersymmetric higher spins we refer the reader to the following. The first Lagrangian description of supersymmetric, massless, higher spins in $4 D$ Minkowski space was done in [27, 28], using components with on-shell supersymmetry. A natural approach to the off-shell formulation is to use the superspace and superfield methods (see e.g. [25, 26]). A superfield description of free supersymmetric massless, higher spin theories was presented for the first time in [29-31] for both Minkowski and AdS spaces. This approach has been further explored in [32-35]. Later studies of free supersymmetric, massless higher spin supermultiplets include [36-40].

\section{Anti-linear transformation of the chiral superfield}

Let's consider a chiral superfield $\Phi$. The most general antilinear transformation one can write is: ${ }^{7}$

$$
\begin{aligned}
\delta_{1} \Phi=\sum_{k=0}^{\infty}\left\{A^{\alpha(k) \dot{\alpha}(k+1)} \overline{\mathrm{D}}_{\dot{\alpha}_{k+1}} \mathrm{D}_{\alpha_{k}} \overline{\mathrm{D}}_{\dot{\alpha}_{k}} \ldots \mathrm{D}_{\alpha_{1}} \overline{\mathrm{D}}_{\dot{\alpha}_{1}} \bar{\Phi}\right. \\
+\Delta^{\alpha(k) \dot{\alpha}(k)} \mathrm{D}_{\alpha_{k}} \overline{\mathrm{D}}_{\dot{\alpha}_{k}} \ldots \mathrm{D}_{\alpha_{1}} \overline{\mathrm{D}}_{\dot{\alpha}_{1}} \bar{\Phi} \\
+\Gamma^{\alpha(k+1) \dot{\alpha}(k)} \mathrm{D}_{\alpha_{k+1}} \overline{\mathrm{D}}^{2} \mathrm{D}_{\alpha_{k}} \overline{\mathrm{D}}_{\dot{\alpha}_{k}} \ldots \mathrm{D}_{\alpha_{1}} \overline{\mathrm{D}}_{\dot{\alpha}_{1}} \bar{\Phi} \\
\left.+E^{\alpha(k) \dot{\alpha}(k)} \overline{\mathrm{D}}^{2} \mathrm{D}_{\alpha_{k}} \overline{\mathrm{D}}_{\dot{\alpha}_{k}} \ldots \mathrm{D}_{\alpha_{1}} \overline{\mathrm{D}}_{\dot{\alpha}_{1}} \bar{\Phi}\right\}
\end{aligned}
$$

The consistency of this transformation with the chiral condition of $\Phi, \overline{\mathrm{D}}_{\dot{\alpha}} \Phi=0$ constraints the parameters of the transformation in the following way:

$$
\begin{aligned}
& \overline{\mathrm{D}}_{\dot{\beta}} A^{\alpha(k) \dot{\alpha}(k+1)}+\frac{1}{(k+1) !} \Delta^{\alpha(k)(\dot{\alpha}(k)} \delta_{\dot{\beta}}^{\left.\dot{\alpha}_{k+1}\right)}=0, \\
& \overline{\mathrm{D}}_{\dot{\beta}} \Delta^{\alpha(k) \dot{\alpha}(k)}=0, \\
& \frac{k+1}{k+2} \Delta^{\alpha(k+1) \dot{\alpha}(k+1)} C_{\dot{\beta} \dot{\alpha}_{k+1}}+\overline{\mathrm{D}}_{\dot{\beta}} \Gamma^{\alpha(k+1) \dot{\alpha}(k)}=0, \\
& A^{\alpha(k+1) \dot{\alpha}(k+2)} C_{\dot{\beta} \dot{\alpha}_{k+2}}+\overline{\mathrm{D}}_{\dot{\beta}} E^{\alpha(k+1) \dot{\alpha}(k+1)}-\frac{1}{(k+1) !} \Gamma^{\alpha(k+1)(\dot{\alpha}(k)} \delta_{\dot{\beta}}^{\left.\dot{\alpha}_{k+1}\right)}=0, \\
& A^{\dot{\alpha}} C_{\dot{\beta} \dot{\alpha}}+\overline{\mathrm{D}}_{\dot{\beta}} E=0 .
\end{aligned}
$$

The solution of the above set of constraints is:

$$
\begin{aligned}
A_{\alpha(k) \dot{\alpha}(k+1)} & =\frac{1}{(k+1) !} \overline{\mathrm{D}}_{\left(\dot{\alpha}_{k+1}\right.} \bar{\xi}_{\alpha(k) \dot{\alpha}(k))}-\overline{\mathrm{D}}^{2} \bar{\ell}_{\alpha(k) \dot{\alpha}(k+1)}, \\
\Delta_{\alpha(k) \dot{\alpha}(k)} & =\overline{\mathrm{D}}^{2} \bar{\xi}_{\alpha(k) \dot{\alpha}(k)} \\
\Gamma_{\alpha(k+1) \dot{\alpha}(k)} & =\frac{k+1}{k+2} \overline{\mathrm{D}}^{\dot{\alpha}_{k+1}} \bar{\xi}_{\alpha(k+1 \dot{\alpha}(k+1)}, \\
E_{\alpha(k) \dot{\alpha}(k)} & =-\bar{\xi}_{\alpha(k) \dot{\alpha}(k)}-\overline{\mathrm{D}}^{\dot{\alpha}_{k+1}} \bar{\ell}_{\alpha(k) \dot{\alpha}(k+1)}
\end{aligned}
$$

\footnotetext{
${ }^{6}$ The supertrace is relevant only to the super-Poincare higher spin supermultiplets because their description requires an additional compensating superfield. For integer superspins this is the real $V_{\alpha(s-1) \dot{\alpha}(s-1)}$ superfield.

${ }^{7}$ We are following the "Superspace" [25] conventions.
} 
where $\bar{\xi}_{\alpha(k) \dot{\alpha}(k)}$ and $\bar{\ell}_{\alpha(k) \dot{\alpha}(k+1)}$ are arbitrary, unconstrained superfields. An observation is that all the $\bar{\ell}_{\alpha(k) \dot{\alpha}(k+1)}$ terms in (3.3a) can be adsorbed by doing a field redefinition of $\bar{\xi}_{\alpha(k) \dot{\alpha}(k)}\left(\bar{\xi}_{\alpha(k) \dot{\alpha}(k)} \rightarrow \bar{\xi}_{\alpha(k) \dot{\alpha}(k)}-\overline{\mathrm{D}}^{\dot{\alpha}_{k+1}} \bar{\ell}_{\alpha(k) \dot{\alpha}(k+1)}\right)$. However, because the $\bar{\xi}_{\alpha(k) \dot{\alpha}(k)}$ and the $\bar{\ell}_{\alpha(k) \dot{\alpha}(k+1)}$ parts of (3.3a) are similar in structure to the terms that appear in the complex conjugate versions of the conformal (2.9) and Poincaré (2.6a) transformations respectively, it will be convenient to consider them separately. The significance of these two cases is related to the fact that conformal integer superspin multiplets have a bigger gauge symmetry (2.9) than the Poincaré integer superspin multiplets (2.6a). Only by further constraining the gauge parameter $\Xi_{\alpha(s-1) \dot{\alpha}(s-1)}$ of the conformal case down to $\Xi_{\alpha(s-1) \dot{\alpha}(s-1)}=\mathrm{D}^{\alpha_{s}} L_{\alpha(s-1) \dot{\alpha}(s-1)}$ one can introduce an appropriate compensating superfield that will break the conformal symmetry to its Poincaré subgroup. It is interesting that this mismatch between the corresponding gauge transformations of conformal and Poincaré higher superspin multiplets appears only for the integer superspin values. In [41] it was demonstrated that for the matter gravitino supermultiplet $[Y=1](3 / 2,1)$ one can relax the Poincaré gauge transformation to match the conformal one, by adding another compensating superfield with an algebraic (no derivatives) transformation law. Recently [20] this mechanism was applied to higher integer superspin supermultiplets. However, this description is non-economical (requires more superfields than it is necessary) and one can always use the algebraic nature of the transformation of the additional compensator in order to remove it. Hence, we will work using the (2.6a) description where the transformations of the conformal and Poincaré supermultiplets differ. For the half-integer superspin multiplets the corresponding gauge transformations between conformal and Poincaré cases are identical. This is can also be seen as the reason why the equivalent analysis for the cubic interaction of the chiral with the half-integer superspin multiplets [18, 21] gave a unigue class of consistent transformations for the chiral superfield.

The most encouraging observation is the similarity between the structure of the gauge parameters that parametrize the transformation of the chiral superfield and the gauge transformations of the conformal or Poincaré integer superspin supermultiplets. This is a hint that indeed we can find cubic interactions of the chiral superfield with the integer superspins.

Therefore, based on the above results we will consider the following two classes of chiral transformations:

1. $\quad \delta_{1} \Phi=\sum_{k=0}^{\infty}\left\{\frac{1}{(k+1) !} \overline{\mathrm{D}}^{\left(\dot{\alpha}_{k+1}\right.} \bar{\xi}^{\alpha(k) \dot{\alpha}(k))} \overline{\mathrm{D}}_{\dot{\alpha}_{k+1}} \mathrm{D}_{\alpha_{k}} \overline{\mathrm{D}}_{\dot{\alpha}_{k}} \ldots \mathrm{D}_{\alpha_{1}} \overline{\mathrm{D}}_{\dot{\alpha}_{1}} \bar{\Phi}\right.$

$$
\begin{aligned}
& +\overline{\mathrm{D}}^{2} \bar{\xi}^{\alpha(k) \dot{\alpha}(k)} \mathrm{D}_{\alpha_{k}} \overline{\mathrm{D}}_{\dot{\alpha}_{k}} \ldots \mathrm{D}_{\alpha_{1}} \overline{\mathrm{D}}_{\dot{\alpha}_{1}} \bar{\Phi} \\
& -\frac{k+1}{k+2} \overline{\mathrm{D}}_{\dot{\alpha}_{k+1}} \bar{\xi}^{\alpha(k+1 \dot{\alpha}(k+1)} \mathrm{D}_{\alpha_{k+1}} \overline{\mathrm{D}}^{2} \mathrm{D}_{\alpha_{k}} \overline{\mathrm{D}}_{\dot{\alpha}_{k}} \ldots \mathrm{D}_{\alpha_{1}} \overline{\mathrm{D}}_{\dot{\alpha}_{1}} \bar{\Phi} \\
& \left.-\bar{\xi}^{\alpha(k) \dot{\alpha}(k)} \overline{\mathrm{D}}^{2} \mathrm{D}_{\alpha_{k}} \overline{\mathrm{D}}_{\dot{\alpha}_{k}} \ldots \mathrm{D}_{\alpha_{1}} \overline{\mathrm{D}}_{\dot{\alpha}_{1}} \bar{\Phi}\right\} \\
& \text { 2. } \quad \delta_{1} \Phi=\sum_{k=0}^{\infty}\left\{\overline{\mathrm{D}}^{2} \bar{\ell}^{\alpha(k) \dot{\alpha}(k+1)} \overline{\mathrm{D}}_{\dot{\alpha}_{k+1}} \mathrm{D}_{\alpha_{k}} \overline{\mathrm{D}}_{\dot{\alpha}_{k}} \ldots \mathrm{D}_{\alpha_{1}} \overline{\mathrm{D}}_{\dot{\alpha}_{1}} \bar{\Phi}\right. \\
& \left.+\overline{\mathrm{D}}_{\dot{\alpha}_{k+1}} \bar{\ell}^{\alpha(k) \dot{\alpha}(k+1)} \overline{\mathrm{D}}^{2} \mathrm{D}_{\alpha_{k}} \overline{\mathrm{D}}_{\dot{\alpha}_{k}} \ldots \mathrm{D}_{\alpha_{1}} \overline{\mathrm{D}}_{\dot{\alpha}_{1}} \bar{\Phi}\right\}
\end{aligned}
$$


We will demonstrate that the first will generate the conformal integer superspin supercurrents and the corresponding interactions with the conformal integer superspin supermultiplets, whereas the second will lead to the Poincaré integer superspin supercurrents and the interactions of matter with Poincaré integer superspin supermultiplets.

\section{Conformal integer superspin supercurrents for free massless chiral}

Let's consider the case of a single, free, massless chiral superfield

$$
S_{0}=\int d^{8} z \bar{\Phi} \Phi
$$

Using (3.4a) and the action above, we can follow the steps of Noether's method, in order to find the corresponding conserved supercurrents. These type of calculations are done onshell (modulo terms which depend on the equations of motion) where the conservation of the (super)current is revealed. In this case the equation of motion takes the form $\overline{\mathrm{D}}^{2} \bar{\Phi}=0$, hence in the variation of the action we can ignore terms that depend on $\overline{\mathrm{D}}^{2} \bar{\Phi}$. Notice that our transformations (3.4a) have a few terms of these type, hence their contribution to the variation of the action can be immediately ignored. The same conclusion can be reached by a different argument which is based on the distinction of the various terms that appear in (3.4a) into two classes. The first one is the class of terms that will generate non-trivial effects. The second one is the class of terms that have trivial contributions which can be absorbed by appropriate field redefinitions. The parts of transformations (3.4a) that depend on $\bar{D}^{2} \bar{\Phi}$ fall into the second class. As an example, consider the $k=0$ part of (3.4a) $\left(-\bar{\xi}+\overline{\mathrm{D}}_{\dot{\alpha}} \bar{\ell}^{\dot{\alpha}}\right) \overline{\mathrm{D}}^{2} \bar{\Phi}$ and calculate its effect in the transformed action: ${ }^{8}$

$$
S_{0}=\int \bar{\Phi} \Phi+g \int\left[\bar{\Phi}\left(-\bar{\xi}+\overline{\mathrm{D}}_{\dot{\alpha}} \bar{\ell}^{\dot{\alpha}}\right) \overline{\mathrm{D}}^{2} \bar{\Phi}+\text { c.c. }\right] .
$$

By doing an integration by part this can be written in the following form

$$
S_{0}=\int \bar{\Phi} \Phi+g \int\left[\overline{\mathrm{D}}^{2}\left\{\bar{\Phi}\left(-\bar{\xi}+\overline{\mathrm{D}}_{\dot{\alpha}} \bar{\ell}^{\dot{\alpha}}\right)\right\} \bar{\Phi}+\text { c.c. }\right]
$$

hence, one can do the following redefinition $\Phi \rightarrow \Phi-g \overline{\mathrm{D}}^{2}\left[\bar{\Phi}\left(-\bar{\xi}+\overline{\mathrm{D}}_{\dot{\alpha}} \bar{\ell}^{\dot{\alpha}}\right)\right]$ and completely absorb the second term, up to order $g$. Similar arguments holds for all terms of (3.4a) that depend on $\bar{D}^{2} \bar{\Phi}$. Their effect in the variation of the action can be counteracted (up to order $g$ ) by redefinitions of the type $\Phi \rightarrow \Phi-$ $g \overline{\mathrm{D}}^{2}\left\{\bar{\Phi} \sum_{k=0}^{\infty}\left[\partial^{(k)} F_{\alpha(k) \dot{\alpha}(k)}+\mathrm{D}^{\alpha_{k+1}} \partial^{(k)} G_{\alpha(k+1) \alpha(k)}\right]\right\}$ for arbitrary superfields $F_{\alpha(k) \dot{\alpha}(k)}$ and $G_{\alpha(k+1) \dot{\alpha}(k)}$. These terms are precisely the terms that are dropped by following the "onshell" approach, so the two arguments are in complete agreement. With that in mind, we use (3.4a) to calculate the variation of $S_{0}$ to be:

$$
\begin{aligned}
\delta_{g} S_{0}=g \sum_{k=0}^{\infty} \int\left[\frac{1}{(k+1) !} \mathrm{D}^{\left(\alpha_{k+1}\right.} \xi^{\alpha(k)) \dot{\alpha}(k)}\left\{(-i)^{k} \Phi \partial^{(k)} \mathrm{D} \Phi\right\}\right. \\
\left.+\mathrm{D}^{2} \xi^{\alpha(k)) \dot{\alpha}(k)}\left\{(-i)^{k} \Phi \partial^{(k)} \Phi\right\}\right]+ \text { c.c. }
\end{aligned}
$$

\footnotetext{
${ }^{8}$ Keeping only terms linear in $g$.
} 
The quantities inside the curly brackets are not uniquely defined because one can consider improvement terms $A_{\alpha(k+1) \dot{\alpha}(k)}$ and $B_{\alpha(k) \dot{\alpha}(k)}$ that satisfy:

$$
\mathrm{D}^{\alpha_{k+1}} A_{\alpha(k+1) \dot{\alpha}(k)}=\mathrm{D}^{2} B_{\alpha(k) \dot{\alpha}(k)} \text { (up to terms that depend on e.o.m). }
$$

A general expression for the improvement terms is

$$
\begin{aligned}
A_{\alpha(k+1) \dot{\alpha}(k)} & =\frac{k+1}{(k+2) !} \mathrm{D}_{\left(\alpha_{k+1}\right.} \zeta_{\alpha(k)) \dot{\alpha}(k)}+\frac{1}{k !} \overline{\mathrm{D}}_{\left(\dot{\alpha}_{k}\right.} \mathrm{D}^{2} \kappa_{\alpha(k+1) \dot{\alpha}(k-1))}+X_{\alpha(k+1) \dot{\alpha}(k)} \\
B_{\alpha(k) \dot{\alpha}(k)} & =\zeta_{\alpha(k) \dot{\alpha}(k)}+\frac{1}{k !} \overline{\mathrm{D}}_{\left(\dot{\alpha}_{k}\right.} \mathrm{D}^{\alpha_{k+1}} \kappa_{\alpha(k+1) \dot{\alpha}(k-1))}+Y_{\alpha(k) \dot{\alpha}(k)}
\end{aligned}
$$

where $\mathrm{D}^{\alpha_{k+1}} X_{\alpha(k+1) \dot{\alpha}(k)}=0$ and $\mathrm{D}^{2} Y_{\alpha(k) \dot{\alpha}(k)}=0$ modulo terms that depend on $\overline{\mathrm{D}}^{2} \bar{\Phi}$. The superfield $X_{\alpha(k+1) \dot{\alpha}(k)}$ may include terms like $\mathrm{D}^{\alpha_{k+2}} P_{\alpha(k+2) \dot{\alpha}(k)}^{(1)}$ or $\mathrm{D}^{2} P_{\alpha(k+1) \dot{\alpha}(k)}^{(2)}$ which identically satisfy $X$ 's constraint due to the algebra of the covariant spinorial derivatives. However, it is important to state that there can be non-trivial solutions which do not fit into this form. An example of this has been demonstrated in [22]. A similar statement holds true for superfield $Y_{\alpha(k) \dot{\alpha}(k)}$. Therefore, equation (4.4) can be written in the following way

$$
\delta_{g} S_{0}=g \sum_{k=0}^{\infty} \int\left[\frac{1}{(k+1) !} \mathrm{D}^{\left(\alpha_{k+1}\right.} \xi^{\alpha(k)) \dot{\alpha}(k)} \mathcal{J}_{\alpha(k+1) \dot{\alpha}(k)}+\mathrm{D}^{2} \xi^{\alpha(k)) \dot{\alpha}(k)} \mathcal{T}_{\alpha(k) \dot{\alpha}(k)}\right]+\text { c.c. }
$$

where

$$
\begin{aligned}
\mathcal{J}_{\alpha(k+1) \dot{\alpha}(k)}= & (-i)^{k} \Phi \partial^{(k)} \mathrm{D} \Phi+\frac{k+1}{(k+2) !} \mathrm{D}_{\left(\alpha_{k+1}\right.} \zeta_{\alpha(k)) \dot{\alpha}(k)} \\
& +\frac{1}{k !} \overline{\mathrm{D}}_{\left(\dot{\alpha}_{k}\right.} \mathrm{D}^{2} \kappa_{\alpha(k+1) \dot{\alpha}(k-1))}+X_{\alpha(k+1) \dot{\alpha}(k)} \\
\mathcal{T}_{\alpha(k) \dot{\alpha}(k)}= & (-i)^{k} \Phi \partial^{(k)} \Phi+\zeta_{\alpha(k) \dot{\alpha}(k)}+\frac{1}{k !} \overline{\mathrm{D}}_{\left(\dot{\alpha}_{k}\right.} \mathrm{D}^{\alpha_{k+1}} \kappa_{\alpha(k+1) \dot{\alpha}(k-1))}+Y_{\alpha(k) \dot{\alpha}(k)}
\end{aligned}
$$

Exploiting the freedom of the unconstrained $\zeta_{\alpha(k) \dot{\alpha}(k)}$ improvement term we can select it appropriately such that $\mathcal{T}_{\alpha(k) \dot{\alpha}(k)}=0$. With this choice, the variation of $S_{0}$ reduces to:

$$
\delta_{g} S_{0}=g \sum_{k=0}^{\infty} \int\left[\frac{1}{(k+1) !} \mathrm{D}^{\left(\alpha_{k+1}\right.} \xi^{\alpha(k)) \dot{\alpha}(k)} \mathcal{J}_{\alpha(k+1) \dot{\alpha}(k)}\right]+\text { c.c. }
$$

with

$$
\begin{aligned}
\mathcal{J}_{\alpha(k+1) \dot{\alpha}(k)}= & \frac{(-i)^{k}}{k+2} \Phi \partial^{(k)} \mathrm{D} \Phi-\frac{k+1}{k+2}(-i)^{k} \mathrm{D} \Phi \partial^{(k)} \Phi+X_{\alpha(k+1) \dot{\alpha}(k)} \\
& -\frac{k+1}{(k+2) !} \mathrm{D}_{\left(\alpha_{k+1}\right.} Y_{\alpha(k)) \dot{\alpha}(k)}+\frac{1}{k !} \overline{\mathrm{D}}_{\left(\dot{\alpha}_{k}\right.} \mathrm{D}^{2} \kappa_{\alpha(k+1) \dot{\alpha}(k-1))} \\
& -\frac{k+1}{k+2} \frac{1}{(k+1) ! k !} \mathrm{D}_{\left(\alpha_{k+1}\right.} \overline{\mathrm{D}}_{\left(\dot{\alpha}_{k}\right.} \mathrm{D}^{\beta} \kappa_{\beta \alpha(k)) \dot{\alpha}(k-1))} .
\end{aligned}
$$


The $Y_{\alpha(k) \dot{\alpha}(k)}$ and $\kappa_{\alpha(k+1) \dot{\alpha}(k)}$ terms of (4.10) can be absorbed by appropriate redefinition of $X_{\alpha(k+1) \dot{\alpha}(k)}$ (they are consistent with $\left.\mathrm{D}^{\alpha_{k+1}} X_{\alpha(k+1) \dot{\alpha}(k)}=0\right)$, hence we can simplify the expression for $\mathcal{J}_{\alpha(k+1) \dot{\alpha}(k)}$ :

$$
\mathcal{J}_{\alpha(k+1) \dot{\alpha}(k)}=\frac{(-i)^{k}}{k+2} \Phi \partial^{(k)} \mathrm{D} \Phi-\frac{k+1}{k+2}(-i)^{k} \mathrm{D} \Phi \partial^{(k)} \Phi+X_{\alpha(k+1) \dot{\alpha}(k)} .
$$

In order to get consistent interactions with conformal integer superspin supermultiplets $\left(\Psi_{\alpha(s) \dot{\alpha}(s-1)}\right)$ we have to consider the full transformation (2.9) and not just a part of it as it appears in (4.9). However we can write ${ }^{9}$

$$
\begin{aligned}
& \delta_{g} S_{0}=g \sum_{k=1}^{\infty} \int\left[\left\{\frac{1}{(k+1) !} \mathrm{D}^{\left(\alpha_{k+1}\right.} \xi^{\alpha(k)) \dot{\alpha}(k)}\right.\right. \\
&\left.\left.+\frac{1}{k !} \overline{\mathrm{D}}^{\left(\dot{\alpha}_{k}\right.} \lambda^{\alpha(k+1) \dot{\alpha}(k-1))}\right\} \mathcal{J}_{\alpha(k+1) \dot{\alpha}(k)}\right]+ \text { c.c. }
\end{aligned}
$$

if and only if $\mathcal{J}_{\alpha(k+1) \dot{\alpha}(k)}$ has the property $\overline{\mathrm{D}}^{\dot{\alpha}_{k}} \mathcal{J}_{\alpha(k+1) \dot{\alpha}(k)}=0$, identically. An encouraging observation towards this direction is that the first term in (4.11) has this property. However, the last two terms of (4.11) do not comply for generic $X_{\alpha(k+1) \dot{\alpha}(k)}$. This is reasonable because both these terms originated from the improvement terms consideration and include a lot of freedom. Hence, we must choose the improvement term $X_{\alpha(k+1) \dot{\alpha}(k)}$ appropriately such that

$$
\begin{aligned}
& \mathrm{D}^{\alpha_{k+1}} X_{\alpha(k+1) \dot{\alpha}(k)}=0 \quad \text { (up to } \overline{\mathrm{D}}^{2} \bar{\Phi} \text { terms), } \\
& \overline{\mathrm{D}}^{\dot{\alpha}_{k}}\left[X_{\alpha(k+1) \dot{\alpha}(k)}-\frac{k+1}{k+2}(-i)^{k} \mathrm{D} \Phi \partial^{(k)} \Phi\right]=0 \quad \text { (identically) . }
\end{aligned}
$$

These two conditions will uniquely fix the improvement term $X_{\alpha(k+1) \dot{\alpha}(k)}$. To find the explicit expression of $X_{\alpha(k+1) \dot{\alpha}(k)}$, let's consider the ansatz

$$
X_{\alpha(k+1) \dot{\alpha}(k)}=\sum_{p=0}^{k} c_{p} \partial^{(p)} \mathrm{D} \Phi \partial^{(k-p)} \Phi
$$

Constraints (4.13a) are equivalent to:

$$
\begin{aligned}
c_{k-p} & =-\frac{p+1}{k-p+1} c_{p}, \quad p=0,1, \ldots, k \\
c_{k-p-1} & =\frac{k-p}{p+1} c_{p}, \quad p=1,2, \ldots, k-2 \\
c_{k-1} & =k c_{0}-\frac{k(k+1)}{k+2}(-i)^{k} .
\end{aligned}
$$

\footnotetext{
${ }^{9}$ Notice that we ignored the $k=0$ term, because it does not correspond to higher spin supermultiplets $(k \geq 1)$ but to the matter gravitino supermultiplet. Although the analysis will go through even in that case, for simplicity we will not include it.
} 
It is straightforward to prove that this system of recursive equations has a solution only for odd values of $k(k=2 l+1, l=0,1,2, \ldots)$

$$
\begin{aligned}
& c_{k}=c_{0}=0, \\
& c_{p}=-\frac{(-i)^{k}}{k+2}(-1)^{p}\left(\begin{array}{l}
k \\
p
\end{array}\right)\left(\begin{array}{l}
k+1 \\
p+1
\end{array}\right), \quad p=1,2, \ldots, k-1, \quad k=2 l+1 .
\end{aligned}
$$

To complete the procedure and get an invariant action we must add to the starting action the following cubic interactions term

$$
S_{I}=-g \sum_{l=0}^{\infty} \int \Psi^{\alpha(2 l+2) \dot{\alpha}(2 l+1)} \mathcal{J}_{\alpha(2 l+2) \dot{\alpha}(2 l+1)}+\text { c.c. }
$$

where the superfield $\Psi_{\alpha(2 l+2) \dot{\alpha}(2 l+1)}$ has a transformation that is dictated by the curly brackets of (4.12). This transformation is of the same kind as (2.9) and therefore the superfield $\Psi_{\alpha(2 l+2) \dot{\alpha}(2 l+1)}$ unambiguously describes the conformal integer superspin $Y=$ $2 l+2(l=0,1, \ldots)$ supermultiplet. The cubic interaction term is generated by the integer superspin supercurrent $\mathcal{J}_{\alpha(2 l+2) \dot{\alpha}(2 l+1)}$ :

$$
\mathcal{J}_{\alpha(2 l+2) \dot{\alpha}(2 l+1)}=\frac{i(-1)^{l}}{2 l+3} \sum_{p=0}^{2 l+1}(-1)^{p}\left(\begin{array}{c}
2 l+1 \\
p
\end{array}\right)\left(\begin{array}{c}
2 l+2 \\
p+1
\end{array}\right) \partial^{(p)} \mathrm{D} \Phi \partial^{(2 l+1-p)} \Phi .
$$

Furthermore, one can check that $\mathcal{J}_{\alpha(2 l+2) \dot{\alpha}(2 l+1)}$ satisfies the following conservation equations:

$$
\mathrm{D}^{\alpha_{2 l+2}} \mathcal{J}_{\alpha(2 l+2) \dot{\alpha}(2 l+1)}=0, \quad \overline{\mathrm{D}}^{\alpha_{2 l+1}} \mathcal{J}_{\alpha(2 l+2) \dot{\alpha}(2 l+1)}=0 .
$$

Expression (4.18) matches the Minkowski superspace limit of the AdS integer superspin supercurrents constructed in [23]. The above supercurrent is bilinear to the chiral superfield which describes free massless fields of spin 0 and $1 / 2$. In [42] it was shown that for such composite objects their proper transformation under conformal symmetry is equivalent to the conservation equation conditions and the on-shell equations of motion of its building blocks. Using similar arguments one can confirm that $\mathcal{J}_{\alpha(2 l+2) \dot{\alpha}(2 l+1)}$ is a superconformal primary with weights $\left(1+\frac{2 l+2}{2}, 1+\frac{2 l+1}{2}\right)$ which are appropriate in order to make the cubic interaction (4.17) superconformally invariant. This may feel unexpected because our starting point, transformations (3.4a), break conformal symmetry because they do not preserve the superconformal primary property of $\Phi$. Nevertheless, the fact that we can find an improvement term that makes the supertrace to vanish and at the same time generate a supercurrent with the appropriate conservation equations is a manifestation of the conformal symmetry of this special case. At the level of the action, the same special improvement terms that canceled the supertrace, recombined the effect of various terms in the transformation in such a way in order to generate cubic interactions that respect conformal symmetry. Obviously, this is not a general feature and highly depends on the properties of the starting action. In this case, $S_{0}$ has conformal symmetry, and for that 
reason we were able to reach the minimal supercurrent multiplet ${ }^{10}$ which resulted to a higher spin supercurrent which satisfies the correct conservation equations in order to be superconformally primary and thus restoring the conformal symmetry.

\section{Poincaré integer superspin supercurrents for free massless chiral}

Now, let's consider the effects of (3.4b) on the single, free, massless chiral action (4.1). We get:

$$
\delta_{g} S_{0}=g \sum_{k=0}^{\infty} \int\left[\mathrm{D}^{2} \ell^{\alpha(k+1) \dot{\alpha}(k)}\left\{(-i)^{k} \Phi \partial^{(k)} \mathrm{D} \Phi\right\}\right]+\text { c.c. }
$$

As mentioned previously, the term inside the curly bracket has the property $\overline{\mathrm{D}}^{\dot{\alpha}_{k}}\left\{(-i)^{k} \Phi \partial^{(k)} \mathrm{D} \Phi\right\}=0$, hence we can rewrite (5.1)

$$
\delta_{g} S_{0}=g \sum_{k=1}^{\infty} \int\left[\left\{\mathrm{D}^{2} \ell^{\alpha(k+1) \dot{\alpha}(k)}+\frac{1}{k !} \overline{\mathrm{D}}^{\left(\dot{\alpha}_{k}\right.} \lambda^{\alpha(k+1) \dot{\alpha}(k-1))}\right\} \mathcal{J}_{\alpha(k+1) \dot{\alpha}(k)}\right]+\text { c.c. }
$$

where

$$
\mathcal{J}_{\alpha(k+1) \dot{\alpha}(k)}=(-i)^{k} \Phi \partial^{(k)} \mathrm{D} \Phi
$$

Following Noether's method we find that this supercurrent generates the following cubic interactions between free massless chiral supermultiplet and the Poincaré integer superspin supermultiplet

$$
S_{I}=-g \sum_{s=2}^{\infty} \int \Psi^{\alpha(s) \dot{\alpha}(s-1)} \mathcal{J}_{\alpha(s) \dot{\alpha}(s-1)}+\text { c.c. }
$$

In contrast with the previous conformal case, the supercurrent (and the cubic interaction) is defined for every positive integer $s$ and not just for the even values. Furthermore, one can prove that $\mathcal{J}_{\alpha(s) \dot{\alpha}(s-1)}$ satisfy the following conservation equations:

$$
\mathrm{D}^{2} \mathcal{J}_{\alpha(s) \dot{\alpha}(s-1)}=0, \quad \overline{\mathrm{D}}^{\dot{\alpha}_{s-1}} \mathcal{J}_{\alpha(s) \dot{\alpha}(s-1)}=0
$$

and crucially $\mathrm{D}^{\alpha_{s}} \mathcal{J}_{\alpha(s) \dot{\alpha}(s-1)} \neq 0$, thus it can not be a primary superfield and it is not related with conformal supercurrent.

\section{Integer superspin supercurrent multiplet beyond free, massless, chiral}

In [22] we investigated the construction of half-integer superspin supercurrent multiplet for a general class of non-linear sigma models of a single chiral superfields, parametrized by an arbitrary Kähler potential $\mathcal{K}(\Phi, \bar{\Phi})$ and a chiral superpotential $\mathcal{W}(\Phi)$. The result was that besides the free, massless case, the arbitrary half-integer superspin supercurrent multiplets

\footnotetext{
${ }^{10}$ This terminology was introduced in [43] and we have used in $[18,21]$ in order to emphasize that these are special cases.
} 
exist only for $\mathcal{K}(\Phi, \bar{\Phi})=\bar{\Phi} \Phi$ with $\mathcal{W}(\Phi)=f \Phi$ or $\mathcal{W}(\Phi)=m \Phi^{2}$, which is consistent with the expectations coming from [45-47]. Therefore, it will be interesting to investigate the existence of arbitrary integer superspin supercurrents for the cases of a free chiral with a linear superpotential or a free massive chiral superfield.

In both cases there is a dimension-full parameter, therefore only the super-Poincaré higher spin supermultiplet is relevant. The general cubic interaction of the chiral with the Poincaré integer superspin supermultiplet $Y=s(2.6 \mathrm{a})$ has the form

$$
S_{I}=\int d^{8} z\left[\Psi^{\alpha(s) \dot{\alpha}(s-1)} \mathcal{J}_{\alpha(s) \dot{\alpha}(s-1)}+\frac{1}{2} V^{\alpha(s-1) \dot{\alpha}(s-1)} \mathcal{T}_{\alpha(s-1) \dot{\alpha}(s-1)}\right]+\text { c.c. }
$$

where $\mathcal{J}_{\alpha(s) \dot{\alpha}(s-1)}$ is the arbitrary integer superspin supercurrent and $\mathcal{T}_{\alpha(s-1) \dot{\alpha}(s-1)}$ is the arbitrary integer superspin supertrace. ${ }^{11}$ Due to the gauge symmetries (2.6a) the supercurrent and the supertrace have to respect the following:

$$
\begin{aligned}
\mathrm{D}^{2} \mathcal{J}_{\alpha(s) \dot{\alpha}(s-1)} & =\frac{1}{s !} \mathrm{D}_{\left(\alpha_{s}\right.} \mathcal{T}_{\alpha(s-1)) \dot{\alpha}(s-1)}, \\
\overline{\mathrm{D}}^{\dot{\alpha}_{s-1}} \mathcal{J}_{\alpha(s) \dot{\alpha}(s-1)} & =0
\end{aligned}
$$

where $\mathcal{T}_{\alpha(s-1) \dot{\alpha}(s-1)}$ is real. In previous section, we demonstrated that for the free, massless chiral case the supertrace vanishes. However if we go beyond that, it is reasonable to expect corrections proportional to the dimension-full parameter that controls the added terms.

\subsection{Free chiral with linear superpotential}

Let's consider the addition of a linear superpotential term in (4.1) controlled by a complex parameter $f$ :

$$
S_{0}=\int d^{8} z \bar{\Phi} \Phi+f \int d^{6} z \Phi+f^{*} \int d^{6} \bar{z} \bar{\Phi}
$$

It is straightforward to show that in this case the supercurrent and supertrace are:

$$
\begin{aligned}
\mathcal{J}_{\alpha(s) \dot{\alpha}(s-1)} & =(-i)^{(s-1)} \Phi \partial^{(s-1)} \mathrm{D} \Phi, \\
\mathcal{T}_{\alpha(s-1) \dot{\alpha}(s-1)} & =(-i)^{(s-1)} f^{*} \partial^{(s-1)} \Phi+(i)^{(s-1)} f \partial^{(s-1)} \bar{\Phi} .
\end{aligned}
$$

The supercurrent does not acquire any modifications and remains the same as in the massless case (5.3). The supercurrent and supertrace can be defined for any $(s \geq 2)$ value of the integer $s$.

\footnotetext{
${ }^{11}$ The term supertrace originates from the cubic interaction of matter supermultiplets with the compensator of Poincaré supergravity (see [26]). We have been using the same terminology for the higher spin version of this type of interactions, meaning the interactions with the compensator of the Poincaré halfinteger superspin supermultiplet. For the case of integer superspin supermultiplets, we will continue to use it in the same spirit. The supertrace generates the cubic interactions with the compensator of the Poincaré integer superspin supermultiplet.
} 


\subsection{Free massive chiral}

Let's consider the addition of a mass term in (4.1)

$$
S_{0}=\int d^{8} z \bar{\Phi} \Phi+m \int d^{6} z \Phi^{2}+m \int d^{6} \bar{z} \bar{\Phi}^{2}
$$

with a real mass parameter $m$. In this case one can show that the integer superspin supercurrent and supertrace exist only for even values of $s(s=2,4,6, \ldots)$ and they are:

$$
\begin{aligned}
& \mathcal{J}_{\alpha(2 l+2) \dot{\alpha}(2 l+1)}=(-1)^{(l+1)} i \Phi \partial^{(2 l+1)} \mathrm{D} \Phi, \\
& \mathcal{T}_{\alpha(2 l+1) \dot{\alpha}(2 l+1)}=(-1)^{(l+1)} i m \bar{\Phi} \partial^{(2 l+1)} \Phi-(-1)^{(l+1)} i m \Phi \partial^{(2 l+1)} \bar{\Phi} .
\end{aligned}
$$

\section{$7 \quad$ Summary and discussion}

In recent work $[18,21]$ it has been shown that linear, higher spin transformations of matter superfields (such as the chiral or the complex linear) exist and are responsible for generating (by following Noether's procedure) consistent cubic interactions between matter multiplets and half integer superspin $(Y=s+1 / 2)$ supermultiplets in terms of half-integer superspin supercurrents. Following this method we were able to reproduce known supercurrents for a massless chiral and find new half-integer superspin supercurrents for a massive chiral. An interesting feature of these new supercurrents was the presence of a selection rule, meaning they can be defined only for odd values of the parameter $s(s=2 l+1)$. These results were later reproduced in [19] following a different approach.

In this work we prove the existence of antilinear, higher spin transformations of the chiral superfield which via Noether's method generate consistent cubic interactions between the chiral and the integer superspin $(Y=s)$ supermultiplets. This is a very interesting feature because antilinear transformations do not appear frequently in physics. For linear transformations we have the intuition of linearized superdiffeomorphisms that give rise to the cubic interactions of the theory to supergravity. However, we do not know an analogue example for antilinear transformations. Using them, we were able to generate new integer superspin supercurrents for the massless chiral and extend them to the case of massive chiral and to the case of a linear superpotential. For some of these theories various selection rules emerge as well.

In detail, the work and results found in this paper are the following. We considered the most general, non-trivial, antilinear transformation of a chiral superfield which is consistent with the chiral constraint. In this case non-trivial means that we can not absorb the transformation by superfield redefinitions. For example, terms of the transformation that depend on the equations of motion (vanish on-shell) once they are introduced in the variation of the action can be ignored because they can be removed by trivial superfield redefinitions. This is equivalent with the usual argument of imposing the on-shell condition when we calculate the supercurrent. It is important to realize that such terms are necessary in order for the transformations to be chiral but once they are used in order to calculate the variation of the action, they correspond to trivial redefinitions and do not contribute to the generation of non-trivial interactions. Thus they can be ignored from the very beginning. 
In this way we find two classes [(3.4a) and (3.4b)] of effective transformations of this type. Considering the effects of such transformations on the free massless chiral action we find:

i. Transformation (3.4a) leads to the construction of cubic interactions (4.17) with the conformal integer superspin supermultiplet $Y=s$ but only for even values of $s$ $(s=2,4, \cdots=2 l+2)$. The interactions are generated by the integer superspin supercurrent $\mathcal{J}_{\alpha(2 l+2) \dot{\alpha}(2 l+1)}$ given by (4.18), which satisfies conservation equations (4.19). This result is consistent with the flat spacetime limit of the results in [23] where the AdS conformal integer superspin supercurrent was constructed by solving the conservation equation.

ii. Transformation (3.4b) leads to the construction of cubic interactions (5.4) with the Poincaré integer superspin supermultiplet $Y=s$ for all values of $s$. The interactions are generated by the supercurrent $\mathcal{J}_{\alpha(s) \dot{\alpha}(s-1)}$ given by (5.3) which satisfies the Poincaré conservation equations (5.5) but not the conformal conservation equations. This is a new supercurrent.

Next, we considered matter theories beyond the simple free massless theory. However based on $[22,45-47]$ the most general theory we can consider for the construction of higher spin supercurrents is the free, massless theory with the addition of a superpotential with linear and quadratic terms. Due to the presence of dimension-full parameters that control these additional terms, we consider only the extention of the massless Poincaré supercurrent. The approach here is to add all possible corrections to the previous Poincare result and demand the conservation equations (6.2a) on-shell. The results we found are the following new supercurrents:

iii. For a free chiral superfield with a linear superpotential, we can construct cubic interactions with the Poincare integer superspin supermultiplet $Y=s$ for all values of $s$. The interactions are generated by a supercurrent $\mathcal{J}_{\alpha(s) \dot{\alpha}(s-1)}$ and a supertrace $\mathcal{T}_{\alpha(s-1) \dot{\alpha}(s-1)}$ given by $(6.4 \mathrm{a})$ with conservation equations $(6.2 \mathrm{a})$.

$i v$. For a free, massive chiral we find cubic interactions with the Poincaré integer superspin supermultiplet $Y=s$, but only for even values of $s(s=2,4, \cdots=2 l+2)$. The supercurrent and supertrace that generate the interaction are given by (6.6a).

In this work, we considered chiral superfields to represent the matter supermultiplets. However, similar constructions can be done for complex linear superfields as is demonstrated in [21]. The fastest and easiest method to extract the corresponding supercurrent multiplets for a complex linear superfield is to use the chiral - complex linear duality. Starting from the supercurrents for the chiral and performing the duality one can get the supercurrents for the complex linear as well as the relative coupling constant which relates the charge of these two matter multiplets for the interaction with higher superspins (see [21]). Additionally, it will be useful to comment that for any of the above higher spin supercurrent multiplets, one can project the corresponding superspace conservation equations to components in order to find the usual spacetime conservation equations and the corresponding higher spin current multiplets. This has been illustrated in detail in [18, 21]. 
Recently, the results of $[18,21]$ have been criticized in [23] as incomplete. For this reason we feel the need to clarify the results obtained in $[18,21]$ in connection with the results obtained here. In $[18,21]$ we considered only consistent, linear transformations of the matter supermultiplets and proved (via Noether's method) that they generate cubic interactions only with half-integer superspin supermultiplets. On the other hand, in [23], using a different method (solving the superspace conservation equations) various AdS cubic interactions have been constructed for both integer and half-integer superspin supermultiplets. The authors concluded that the results regarding integer superspin interactions have been overlooked in $[18,21]$. We communicated the context of our work to the authors and we informed them that the interactions with the integer superspin supermultiplets will originate from a consideration of antilinear transformations, as demonstrated in this work. An updated version of [23], included this argument and an indicative expression of such antilinear transformations was added.

\section{Acknowledgments}

The authors acknowledge S. M. Kuzenko for the constructive criticism. The research of I. L. B. was supported in parts by Russian Ministry of Education and Science, project No. 3.1386.2017. He is also grateful to RFBR grant, project No. 18-02-00153 for partial support. The research of S. J. G. and K. K. is supported by the endowment of the Ford Foundation Professorship of Physics at Brown University. Also this work was partially supported by the U.S. National Science Foundation grant PHY-1315155.

Open Access. This article is distributed under the terms of the Creative Commons Attribution License (CC-BY 4.0), which permits any use, distribution and reproduction in any medium, provided the original author(s) and source are credited.

\section{References}

[1] F.A. Berends, G.J.H. Burgers and H. van Dam, Explicit construction of conserved currents for massless fields of arbitrary spin, Nucl. Phys. B 271 (1986) 429 [INSPIRE].

[2] D. Anselmi, Higher spin current multiplets in operator product expansions, Class. Quant. Grav. 17 (2000) 1383 [hep-th/9906167] [INSPIRE].

[3] M.A. Vasiliev, Higher spin gauge theories: Star product and AdS space, in The many faces of the superworld, M. Shifman ed., World Scientific, Singapore (2000), hep-th/9910096 [INSPIRE].

[4] S.E. Konstein, M.A. Vasiliev and V.N. Zaikin, Conformal higher spin currents in any dimension and AdS/CFT correspondence, JHEP 12 (2000) 018 [hep-th/0010239] [INSPIRE].

[5] O.A. Gelfond, E.D. Skvortsov and M.A. Vasiliev, Higher spin conformal currents in Minkowski space, Theor. Math. Phys. 154 (2008) 294 [hep-th/0601106] [INSPIRE].

[6] X. Bekaert, E. Joung and J. Mourad, On higher spin interactions with matter, JHEP 05 (2009) 126 [arXiv:0903.3338] [INSPIRE]. 
[7] X. Bekaert and E. Meunier, Higher spin interactions with scalar matter on constant curvature spacetimes: conserved current and cubic coupling generating functions, JHEP $\mathbf{1 1}$ (2010) 116 [arXiv: 1007.4384] [INSPIRE].

[8] X. Bekaert, E. Joung and J. Mourad, Effective action in a higher-spin background, JHEP 02 (2011) 048 [arXiv: 1012.2103] [INSPIRE].

[9] C. Sleight and M. Taronna, Higher spin interactions from conformal field theory: the complete cubic couplings, Phys. Rev. Lett. 116 (2016) 181602 [arXiv:1603.00022] [INSPIRE].

[10] O.A. Gelfond and M.A. Vasiliev, Current interactions from the one-form sector of nonlinear higher-spin equations, Nucl. Phys. B 931 (2018) 383 [arXiv:1706.03718] [INSPIRE].

[11] E.S. Fradkin and R.R. Metsaev, A cubic interaction of totally symmetric massless representations of the Lorentz group in arbitrary dimensions, Class. Quant. Grav. 8 (1991) L89 [INSPIRE].

[12] R.R. Metsaev, Note on the cubic interaction of massless representations of the Poincaré group in D $=5$ space-time, Class. Quant. Grav. 10 (1993) L39 [INSPIRE].

[13] R.R. Metsaev, Cubic interaction vertices of totally symmetric and mixed symmetry massless representations of the Poincaré group in D=6 space-time, Phys. Lett. B 309 (1993) 39 [INSPIRE].

[14] R.R. Metsaev, Generating function for cubic interaction vertices of higher spin fields in any dimension, Mod. Phys. Lett. A 8 (1993) 2413 [INSPIRE].

[15] R.R. Metsaev, Cubic interaction vertices of massive and massless higher spin fields, Nucl. Phys. B 759 (2006) 147 [hep-th/0512342] [INSPIRE].

[16] R.R. Metsaev, Cubic interaction vertices for fermionic and bosonic arbitrary spin fields, Nucl. Phys. B 859 (2012) 13 [arXiv: 0712.3526] [INSPIRE].

[17] S.M. Kuzenko, R. Manvelyan and S. Theisen, Off-shell superconformal higher spin multiplets in four dimensions, JHEP 07 (2017) 034 [arXiv: 1701.00682] [INSPIRE].

[18] I.L. Buchbinder, S.J. Gates Jr. and K. Koutrolikos, Higher spin superfield interactions with the chiral supermultiplet: conserved supercurrents and cubic vertices, Universe 4 (2018) 6 [arXiv: 1708.06262] [INSPIRE].

[19] J. Hutomo and S.M. Kuzenko, Non-conformal higher spin supercurrents, Phys. Lett. B 778 (2018) 242 [arXiv: 1710.10837] [INSPIRE].

[20] J. Hutomo and S.M. Kuzenko, The massless integer superspin multiplets revisited, JHEP 02 (2018) 137 [arXiv:1711.11364] [INSPIRE].

[21] K. Koutrolikos, P. Kočí and R. von Unge, Higher spin superfield interactions with complex linear supermultiplet: conserved supercurrents and cubic vertices, JHEP 03 (2018) 119 [arXiv: 1712.05150] [INSPIRE].

[22] I.L. Buchbinder, S.J. Gates Jr. and K. Koutrolikos, Interaction of supersymmetric nonlinear $\sigma$-models with external higher spin superfields via higher spin supercurrents, JHEP 05 (2018) 204 [arXiv: 1804.08539] [INSPIRE].

[23] E.I. Buchbinder, J. Hutomo and S.M. Kuzenko, Higher spin supercurrents in Anti-de Sitter space, JHEP 09 (2018) 027 [arXiv:1805.08055] [INSPIRE]. 
[24] I.L. Buchbinder, S.J. Gates Jr. and K. Koutrolikos, Conserved higher spin supercurrents for arbitrary spin massless supermultiplets and higher spin superfield cubic interactions, JHEP 08 (2018) 055 [arXiv: 1805.04413] [INSPIRE].

[25] S.J. Gates Jr., M.T. Grisaru, M. Roček and W. Siegel, Superspace or one thousand and one lessons in supersymmetry, Front. Phys. 58 (1983) 1 [hep-th/0108200] [INSPIRE].

[26] I.L. Buchbinder and S.M. Kuzenko, Ideas and methods of supersymmetry and supergravity: or a walk through superspace, IOP, Bristol U.K. (1998).

[27] T. Curtright, Massless field supermultiplets with arbitrary spin, Phys. Lett. B 85 (1979) 219.

[28] M.A. Vasiliev, 'Gauge' form of description of massless fields with arbitrary spin (in Russian), Yad. Fiz. 32 (1980) 855 [Sov. J. Nucl. Phys. 32 (1980) 439] [INSPIRE].

[29] S.M. Kuzenko, A.G. Sibiryakov and V.V. Postnikov, Massless gauge superfields of higher half integer superspins, JETP Lett. 57 (1993) 534 [INSPIRE].

[30] S.M. Kuzenko and A.G. Sibiryakov, Massless gauge superfields of higher integer superspins, JETP Lett. 57 (1993) 539 [INSPIRE].

[31] S.M. Kuzenko and A.G. Sibiryakov, Free massless higher superspin superfields on the anti-de Sitter superspace, Phys. Atom. Nucl. 57 (1994) 1257 [Yad. Fiz. 57 (1994) 1326] [arXiv: 1112.4612] [INSPIRE].

[32] I.L. Buchbinder, S.M. Kuzenko and A.G. Sibiryakov, Quantization of higher spin superfields in the anti-de Sitter superspace, Phys. Lett. B 352 (1995) 29 [hep-th/9502148] [INSPIRE].

[33] S.J. Gates Jr., S.M. Kuzenko and A.G. Sibiryakov, $N=2$ supersymmetry of higher superspin massless theories, Phys. Lett. B 412 (1997) 59 [hep-th/9609141] [INSPIRE].

[34] S.J. Gates Jr., S.M. Kuzenko and A.G. Sibiryakov, Towards a unified theory of massless superfields of all superspins, Phys. Lett. B 394 (1997) 343 [hep-th/9611193] [INSPIRE].

[35] S.J. Gates Jr. and S.M. Kuzenko, $4 D, N=1$ higher spin gauge superfields and quantized twistors, JHEP 10 (2005) 008 [hep-th/0506255] [INSPIRE].

[36] S.J. Gates Jr. and K. Koutrolikos, On $4 D, \mathcal{N}=1$ massless gauge superfields of arbitrary superhelicity, JHEP 06 (2014) 098.

[37] S.J. Gates Jr. and K. Koutrolikos, On $4 D, \mathcal{N}=1$ massless gauge superfields of higher superspin: half-odd-integer case, arXiv:1310.7386 [INSPIRE].

[38] S.J. Gates Jr. and K. Koutrolikos, On $4 D, \mathcal{N}=1$ massless gauge superfields of arbitrary superhelicity, JHEP 06 (2014) 098 [arXiv:1310.7385] [INSPIRE].

[39] I.L. Buchbinder and K. Koutrolikos, BRST analysis of the supersymmetric higher spin field models, JHEP 12 (2015) 106 [arXiv:1510.06569] [INSPIRE].

[40] S.J. Gates Jr. and K. Koutrolikos, From Diophantus to supergravity and massless higher spin multiplets, JHEP 11 (2017) 063 [arXiv:1707.00194] [INSPIRE].

[41] S.J. Gates Jr. and W. Siegel, $(3 / 2,1)$ superfield of $O(2)$ supergravity, Nucl. Phys. B 164 (1980) 484 [INSPIRE].

[42] N.S. Craigie, V.K. Dobrev and I.T. Todorov, Conformally covariant composite operators in quantum chromodynamics, Annals Phys. 159 (1985) 411 [INSPIRE].

[43] M. Magro, I. Sachs and S. Wolf, Superfield Noether procedure, Annals Phys. 298 (2002) 123 [hep-th/0110131] [INSPIRE]. 
[44] S. Weinberg, The quantum theory of fields. Volume 1: foundations, Cambridge University pRess, Cambridge U.K. (2005).

[45] S.R. Coleman and J. Mandula, All possible symmetries of the s matrix, Phys. Rev. 159 (1967) 1251 [INSPIRE].

[46] R. Haag, J.T. Lopuszanski and M. Sohnius, All possible generators of supersymmetries of the s matrix, Nucl. Phys. B 88 (1975) 257 [INSPIRE].

[47] J. Maldacena and A. Zhiboedov, Constraining conformal field theories with a higher spin symmetry, J. Phys. A 46 (2013) 214011 [arXiv:1112.1016] [INSPIRE]. 